DRAFT VERSION JUNE 23, 2018

Preprint typeset using LTEX style emulateapj v. 5/2/11

\title{
SIMULTANEOUS X-RAY AND RADIO OBSERVATIONS OF ROTATING RADIO TRANSIENT J1819-1458
}

\author{
J. J. Miller ${ }^{1}$, M. A. MCLaughlin ${ }^{1,2}$, N. ReA ${ }^{3}$, K. LAZAridis ${ }^{4}$, E. F. KeAne ${ }^{4}$, M. Kramer ${ }^{4,5}$, A. LYNE $^{5}$ \\ Draft version June 23, 2018
}

\begin{abstract}
We present the results of simultaneous radio and X-ray observations of PSR J1819-1458. Our 94-ks XMMNewton observation of the high magnetic field $\left(\sim 5 \times 10^{13} \mathrm{G}\right)$ pulsar reveals a blackbody spectrum $(\mathrm{kT} \sim 130 \mathrm{eV})$ with a broad absorption feature, possibly composed of two lines at $\sim 1.0$ and $\sim 1.3 \mathrm{keV}$. We performed a correlation analysis of the X-ray photons with radio pulses detected in 16.2 hours of simultaneous observations at 1-2 GHz with the Green Bank, Effelsberg, and Parkes telescopes, respectively. Both the detected X-ray photons and radio pulses appear to be randomly distributed in time. We find tentative evidence for a correlation between the detected radio pulses and X-ray photons on timescales of less than 10 pulsar spin periods, with the probability of this occurring by chance being $0.46 \%$. This suggests that the physical process producing the radio pulses may also heat the polar-cap.

Subject headings: pulsars: individual (J1819-1458) — radio continuum: stars — stars: neutron — X-rays: stars
\end{abstract}

\section{INTRODUCTION}

There are over 2000 known pulsar 6 , with roughly 70 of these labeled as Rotating Radio Transients (RRATs) 77; see Keane \& McLaughlin (2011) for a recent review. The single pulses of RRATs have similar widths and intensities to single pulses of other pulsars, but despite an underlying periodicity at the neutron star's rotational period, radio pulses are sporadically detected. It is unclear why the emission of these objects is so sporadic, and numerous theories have been put forward which rely on both internal factors, such as RRATs may be dying or extreme nulling pulsars (Zhang et al. 2007), or external factors such as modulation of the emitted pulses from a radiation belt similar to planetary magnetospheres (Luo \& Melrose 2007) or disturbances from the pulsar's asteroid belt (Cordes \& Shannon 2008).

PSR J1819-1458 has a spin period of $P=4.26$ s, with a pulse detected roughly every three minutes in Parkes observations above a flux density of $S=0.6 \mathrm{mJy}$ at $1.4 \mathrm{GHz}$ (McLaughlin et al. 2006). It has a characteristic age of $\tau_{c}=$ $117 \mathrm{kyr}$, a spin-down luminosity of $\dot{E}_{\text {rot }}=3 \times 10^{32} \mathrm{ergs} \mathrm{s}^{-1}$, and a high inferred surface magnetic field strength of $B=5 \times$ $10^{13} \mathrm{G}$ at the magnetic equator (see, e.g., Lorimer \& Kramer (2005) for a definition of these terms). The distance estimate from its dispersion measure (DM) of $196.0 \pm 0.4 \mathrm{pc} \mathrm{cm}^{-3}$ (Esamdin et al. 2008) and the Galactic electron density model of Cordes \& Lazio (2002) is $3.6 \mathrm{kpc}$ with considerable (at least $25 \%$ ) uncertainty.

A previous 43-ks observation of PSR J1819-1458 by XMM-Newton (McLaughlin et al. 2007) found best-fit spectral models with neutral hydrogen column densities

\footnotetext{
${ }^{1}$ Department of Physics, West Virginia University, Morgantown, WV 26506.

2 Also adjunct at National Radio Astronomy Observatory, Green Bank, WV 24944

${ }^{3}$ Institut de Ciències de l'Espai (IEEC-CSIC) Campus UAB, Fac. de Ciències, Torre C5, parell, 2a planta 08193 Barcelona, Spain

${ }^{4}$ Max Planck Institut für Radioastronomie, Auf dem Hügel 69, 53121 Bonn, Germany

${ }^{5}$ Jodrell Bank Centre for Astrophysics, School of Physics and Astronomy, University of Manchester, Manchester M13 9PL, UK

${ }^{6} \mathrm{http}: / /$ www.atnf.csiro.au/people/pulsar/psrcat/

${ }^{7} \mathrm{http}: / /$ www.as.wvu.edu/ pulsar/rratalog
}

$N_{\mathrm{H}} \sim 7 \times 10^{21} \mathrm{~cm}^{-2}$, temperatures near $k T \sim 0.14 \mathrm{keV}$, a single absorption line near $\sim 1 \mathrm{keV}$, and unabsorbed fluxes $\sim 2 \times 10^{-12} \mathrm{ergs} \mathrm{cm}^{-2} \mathrm{~s}^{-1}(0.3-5 \mathrm{keV})$, which yield a blackbody emission radius (at infinity assuming a $3.6 \mathrm{kpc}$ distance) of $R=8_{-3}^{+7} \mathrm{~km}$. This temperature is expected for a $10^{4}-10^{5}$ year-old cooling neutron star's emission (Yakovlev \& Pethick 2004), generally in agreement with PSR J1819-1458's age. The unabsorbed fluxes yield luminosities which exceed the spin-down luminosity of PSR J1819-1458 by

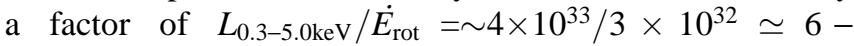
18, depending on the spectral model, which is possible given the thermal origin of the X-ray emission. The results reported by McLaughlin et al. (2007) are consistent with both a chance Chandra observation of PSR J1819-1458 (Reynolds et al. 2006) as well as deeper Chandra observations of PSR J1819-1458 (Rea et al. 2009; Camero-Arranz et al. 2012). The absorption line seen with XMM-Newton by McLaughlin et al. (2007) was confirmed with Chandra by Rea et al. (2009), which rules out instrumentation as the cause. The latter Chandra observations also revealed a bright pulsar wind nebula around PSR J1819-1458, with an inferred X-ray efficiency of $\eta_{X}=L_{\text {pwn:0.5-8.0keV }} / \dot{E}_{\text {rot }}=$ $6.0 \times 10^{31} / 3 \times 10^{32} \simeq 0.2$.

Several spectral models can be used to explain the absorption in the spectrum of PSR J1819-1458. Possible explanations are elements in the interstellar medium (ISM), elements in the neutron star's atmosphere, or cyclotron absorption. A cyclotron proton resonant scattering model yields the magnetic field strength $B_{\mathrm{cy}}=1.6 E_{\mathrm{cy}}(\mathrm{keV}) / y_{\mathrm{G}} 10^{14} \mathrm{G}$, where $E_{\mathrm{cy}}$ is the cyclotron proton energy, and $y_{\mathrm{G}}=\left(1-2 G M / c^{2} R\right)^{1 / 2}$ is the gravitational redshift factor $(\sim 0.77$ using a canonical neutron star mass $M=1.4 M_{\odot}$, and neutron star radius $R=10 \mathrm{~km}$ ). If the cyclotron resonance was due to electrons and not protons, the inferred magnetic field strength would be $m_{p} / m_{e}=1.8 \times 10^{3}$ times weaker, making proton cyclotron resonance more likely due to the high inferred surface magnetic field strength of PSR J1819-1458.

Absorption lines have been observed in several other isolated neutron stars. These include some X-ray Isolated 
Neutron Stars (Hohle et al. (2012); see Turolla (2009) and Kaplan \& van Kerkwiik (2011) for recent reviews), which have absorption lines reported at lower energies $(300-700 \mathrm{eV})$ than those observed for PSR J1819-1458. Furthermore, another rotation-driven pulsar, PSR J1740+1000, has been shown to have an absorption feature around $600 \mathrm{eV}$ (Kargaltsev et al. 2012). It is unclear why some neutron stars exhibit absorption and others do not, with various explanations offered for these absorption lines, e.g. proton cyclotron resonances and atomic transitions in light elements (Turolla 2009; Kaplan \& van Kerkwiik 2011).

While spectral observations are important for probing the pulsar environment, X-ray timing observations can also be useful to learn about emission mechanisms, especially when combined with synchronous radio observations. If radio pulses are correlated with X-ray photons, then a combined mechanism could be responsible for radio and high-energy emission. Such tests have been done to correlate radio giant pulses from the Crab pulsar pulses with non-thermal X-ray and gamma-ray photons. No correlation was found in these studies (Bilous et al. 2011) but a correlation was found between radio giant pulses and optical emission (Shearer et al. 2003), suggesting an overall increase in particle density could be responsible for the giant pulses. In the case of RRATs, Zhang et al. (2007) suggest that we should expect an increase in both non-thermal and thermal X-ray emission close to radio pulse detection times if their sporadicity is due to their reactivation model. This model suggests that the pulsar is only active when the conditions in its magnetosphere allow for pair production which instigates coherent radio emission that results in non-thermal X-ray photons and thermal emission from polar-cap heating. If the pulsar is always active and the sporadicity is due to radio emission direction reversal, however, then the non-thermal and polar-cap heating will always be present and we should therefore not see an increase in X-ray emission close to radio pulse detection times.

We were awarded $94 \mathrm{ks}$ of XMM-Newton time to improve the accuracy of the spectral parameters, determine the origin of the absorption lines, search for evidence of a non-thermal power-law structure in the spectrum, and explore whether the $\mathrm{X}$-ray and radio emission is correlated. We were also awarded time on the Robert C. Byrd Green Bank Telescope (GBT), the Parkes radio telescope, and the Effelsberg radio telescope for simultaneous radio observations. We report here on the results of these observations. In Section 2 we describe the X-ray properties of PSR J1819-1458, quantifying absorption features and the possibility of a power-law tail. In Section 3 we describe the star's radio properties. In Section 4 we compare the observed profile at both wavelengths and present the correlation of pulse arrival times. Finally, we draw some conclusions in Section 5

\section{X-RAY OBSERVATIONS AND ANALYSIS}

We observed PSR J1819-1458 with XMM-Newton for $94 \mathrm{ks}$ on 31 March 2008. These data were taken with EPIC-PN in Full Frame mode and the two MOS with the central CCD in Small Window mode, as was done by McLaughlin et al. (2007). The time resolutions of the EPIC-PN in Full Frame mode and two MOS CCDs in Small Window mode are $73.4 \mathrm{~ms}$ and $0.3 \mathrm{~s}$, respectively. PSR J1819-1458 appeared as a point source with the following J2000 coordinates: right ascension $\alpha=18^{\mathrm{h}} 19^{\mathrm{m}} 34^{\mathrm{s}}$ and declination $\delta=-14^{\circ} 58^{\prime} 04^{\prime \prime}\left(4^{\prime \prime}\right.$ error in each coordinate, where all the errors in this paper are stated at the $1 \sigma$ con- fidence level), consistent with previous X-ray observations and the position derived from radio timing. We could not distinguish the $\sim 5.5^{\prime \prime}$ extended emission region detected by Rea et al. (2009) with Chandra, which has a spatial resolution of $\sim 0.5^{\prime \prime}$ (Weisskopf et al. 2002; Garmire et al. 2003) compared to the $\sim 6^{\prime \prime}$ spatial resolution of XMM-Newton (Watson et al. 2009).

The timing and spectral analyses were done using the XMM-Newton Scientific Analysis System (SAS) tool 18 , version 12.7.0. The Current Calibration File (CCF) was built using the cifbuild command on the SAS tools website?, using the observation date 2008-03-31T14:06:38. In order to exclude events not associated with the pulsar, e.g. solar flares, we defined good time intervals (GTIs) by binning all of the PN and MOS detection times, as well as the detection times from within a $20^{\prime \prime}$ circular radius centered on the source position, into ten-second intervals. We then identified time intervals with excessive photon counts which were not confined to the source region, as determined by visual inspection (areas dominated by non-zero baselines and count rates greater than 100 counts per 10 second time intervals on the PN detector), and excluded those time intervals from the GTIs and our further analysis. Multiple GTI ranges were tested for both timing and spectral analysis. Our analysis resulted in three GTIs for each of the PN, MOS1, and MOS2 detectors. This excluded large bursts at the beginning and end of the observation which were not confined to the source regions; these GTIs span $68.6 \mathrm{ks}$ (19 hrs) from MJD 54556.8 to MJD 54557.6 with each detector having three small interruptions, each spanning 3.5-15.6 seconds, as shown in Table 1. The GTI and photon arrival times were barycentered to the center of the solar system using the XMM analysis tool barycen and the X-ray derived position.

\subsection{Timing Analysis}

Our time resolution is sufficient for studying the pulse profile because of the long period of the pulsar. For timing analysis we included all PN and MOS events within the GTIs satisfying a PATTERN $\leq 12$ requirement (i.e. allowing for single, double, triple, and quadruple events). To ensure extraction of at least $90 \%$ of the source photons, we chose a $20^{\prime \prime}$ circular radius centered on the source position in the data. We also extracted background counts from four nearby $20^{\prime \prime}$ circular region free of point sources and on the same central $\mathrm{CCD}$ as the source region to measure the average background rate. The photon arrival times were folded with the radio timing ephemeris of PSR J1819-1458 using TEMPO10. The data were folded for a combination of 99 trial values of the number of pulse phase bins (2-100 bins), 1969 values of minimum energy $E_{\min }(0.155-9.995 \mathrm{keV})$, and 1969 values of maximum energy $E_{\max }(0.160-10 \mathrm{keV})$, creating $\sim 384$ million profiles. For each trial number of phase bins, value of $E_{\min }$, and value of $E_{\max }$, a $\chi^{2}$ value was calculated for a fit of the folded profile to a flat (i.e. random) distribution. The background rate subtracted X-ray profile with the lowest probability of being drawn from a flat distribution, $P=10^{-52.1}$, has ten phase bins, $E_{\min }=0.5 \mathrm{keV}$, and $E_{\max }=2.6 \mathrm{keV}$ and is shown in Figure 1 Of the 6630 total PN photons within the $20^{\prime \prime}$ radius, 5692 fall within this energy range.

\footnotetext{
${ }^{8} \mathrm{http}: / / \mathrm{xmm} . \mathrm{esa} \cdot \mathrm{int} / \mathrm{sas} /$

${ }^{9} \mathrm{http}: / / \mathrm{xmm}$.vilspa.esa.es/external/xmm_sw_cal/calib/cifbuild.shtml

$10 \mathrm{http}: / /$ www.atnf.csiro.au/research/pulsar/tempo/
} 
A sinusoid was fit to the X-ray profile to determine the peak using a least-squares fitting routine. When adding a second-order sinusoid to the fit, $f(t)=A_{1} \cos \left(2 \pi\left(x-\phi_{0}\right)\right)+$ $A_{2} \cos \left(4 \pi\left(x-\phi_{0}\right)\right)$, the reduced $\chi^{2}$ is decreased from 2.3 to 1.0. Similarly, fitting a Gaussian function produces a fit with reduced $\chi^{2}=1.0$. The phase of the peaks of both the double sinusoid and the Gaussian function was $0.02 \pm 0.01$, where phase zero is the peak of the radio pulse profile. These fits are also shown as the dotted and dashed line in Figure 1, respectively.

The X-ray pulse profile has a $0.5-2.6 \mathrm{keV}$ intrinsic pulsed fraction, defined as $\left(F_{\max }-F_{\min }\right) /\left(F_{\max }+F_{\min }\right)$, where $F_{\max }$ and $F_{\min }$ are the minimum and maximum background-corrected counts of the X-ray pulse profile, of $(33.9 \pm 0.9) \%$, using ten bins and assuming Poisson (i.e. $\sqrt{N}$ ) errors. Previous background-corrected pulsed fractions reported for PSR J1819-1458 are $(34 \pm 6) \%,(28 \pm 7) \%$, and $(49 \pm 10) \%$ for the $0.3-5 \mathrm{keV}, 0.3-1 \mathrm{keV}$, and $1-5 \mathrm{keV}$ energy ranges, respectively (McLaughlin et al. 2007), and $(37 \pm 3) \%$ for the 0.3-5 keV energy range (Rea et al. 2009). Pulsed fractions measured within the same energy ranges with our data set yields $(31.0 \pm 0.8) \%,(30 \pm 1) \%$, and $(49 \pm 1) \%$ for $0.3-5 \mathrm{keV}$, $0.3-1 \mathrm{keV}$, and $1-5 \mathrm{keV}$, respectively.

\subsection{Spectral Analysis}

For spectral analysis, we selected photons from the PN detectors with a more stringent PATTERN $\leq 4$ requirement (i.e. allowing for single and double events), as the background will affect results more significantly. As in the timing analysis, we extracted the source photons from within a $20^{\prime \prime}$ circular radius centered on the source position, which yielded 6974 total events in a $0.5-2.0 \mathrm{keV}$ energy range. We also extracted background counts from four $20^{\prime \prime}$ circular regions centered on off-source positions free of point sources and on the same central CCD as the source region. The spectrum was then rebinned so that there were at least 30 counts per spectral bin so that we could use the $\chi^{2}$ statistic 1 . Additionally, we similarly processed the data from McLaughlin et al. (2007) and added the two observations together with the XSPEC command mathpha with a Gaussian error propagation method to create the spectrum shown in Figure 2. We also processed the MOS1 and MOS2 detections from the observation as well as from McLaughlin et al. (2007) also shown in Figure2. We will only discuss PN spectral analysis hereafter, but both of our MOS spectra model fits are in agreement with the PN spectral analysis.

We restricted the energy range of our spectral fitting to $0.5-2.0 \mathrm{keV}$. This is narrower than that used for timing as at higher energies, the spectrum count rates were comparable to the background region count rates. We were unable to fit a spectral model with $\chi^{2}<2$ without addressing a feature in the residuals of the fits near $\sim 0.5 \mathrm{keV}$. McLaughlin et al. (2007) ignored the $0.5 \mathrm{keV}$ feature by excluding the $0.50-0.53 \mathrm{keV}$ energy range from their spectral fitting, but mentioned that an underabundance of oxygen could explain it. We found that the oxygen edge in the XSPEC model vphabs fit our $\sim 0.5 \mathrm{keV}$ feature well and included it as well as the $0.50-0.53 \mathrm{keV}$ energy range in all of our model fits. Solar abundances from Lodders (2003) were assumed for elements other than Neon and Oxygen. We also investigated fitting our spectral models to energy ranges above $2.0 \mathrm{keV}$, where it looked like a pos-

\footnotetext{
${ }^{11}$ https://heasarc.gsfc.nasa.gov/xanadu/xspec/manual/manual.html
}

sible power-law tail may have been present, but attempts to fit the background dominated portion of the spectrum yielded unacceptable $\chi^{2}$ values.

Modeling the blackbody spectrum without fitting for the $1.0 \mathrm{keV}$ feature results in $\chi^{2} \sim 1.4$. Adding an absorption model around $1.0 \mathrm{keV}$, modeled as either an empirical Gaussian absorption or as cyclotron absorption, yields a better fit, $\chi^{2} \sim 1.2$ (see Table 2). Using an underabundance of neon to explain this feature as was done by McLaughlin et al. (2007) does not yield as good a fit as either the Gaussian or cyclotron absorption. While it is possible that the spectrum could also consist of two blackbody components, cooler emission from the surface along with a smaller hotspot, fitting yielded $\chi^{2}=1.19$ but with large blackbody emission radii errors, (see Table 2). Furthermore, the residuals suggest a second feature around $1.3 \mathrm{keV}$, so we tried to add another Gaussian absorption line to the model, resulting in $\chi^{2}=1.09$. We ran Monte Carlo simulations to assess the significance of the addition of the second absorption feature (see Rea et al. (2005) for further details), and found a significance of $\sim 3 \sigma$ for its addition to the continuum plus one feature model, i.e. $>99 \%$ likelihood of two absorption lines rather than just one. The two-line model is then preferred at a $3 \sigma$ significance, with $\chi^{2}=1.09$ (see Table 2). We did not include the two cyclotron absorption model even though it fit equally well as the others because the two energies are not harmonically related. We also tested XSPEC neutron star atmosphere model nsa which yielded parameters in agreement with those found in Table 2. We performed a phase-resolved analysis, dividing the observation in the onpulse spectra (0.0-0.25 and $0.75-1.0$ pulse phase in Figure 1) and off-pulse spectra ( $0.25-0.75$ pulse phase in Figure 1). Results of the spectral fits to the on- and off-pulse spectra agreed with the parameters fit to the phase-integrated spectra within the parameter uncertainties.

The Leiden/Argentine/Bonn Survey of Galactic HI map (Kalberla et al. 2005) and Dickey and Lockman HI in the Galaxy map (Dickey \& Lockman 1990) quote the total hydrogen column density along the line of sight of PSR J1819-1458 as $1.25 \times 10^{22} \mathrm{~cm}^{-2}$ and $1.64 \times 10^{22} \mathrm{~cm}^{-2}$, respectively, using a weighted average of all points within one degree of PSR J1819-1458. Since the maps represent column densities along the entire line of sight including hydrogen beyond the pulsar, it is reassuring that the hydrogen column densities in Table 2 are generally less than the map measurements. He et al. (2013) found an empirical relationship between $N_{\mathrm{H}}$ and DM for radio pulsars of $N_{\mathrm{H}}\left(10^{20} \mathrm{~cm}^{-2}\right)$ $=0.30_{-0.09}^{+0.13} \mathrm{DM}\left(\mathrm{pc} \mathrm{cm}^{-3}\right)$, which implies an average radio pulsar ionization rate of $10_{-3}^{+4} \%$. When applied to J1819-1458, this relation implies $N_{\mathrm{H}}=0.30_{-0.09}^{+0.13} \times 196.0 \pm 0.4 \mathrm{pc} \mathrm{cm}^{-3}=$ $0.6_{-0.2}^{+0.3} \times 10^{22} \mathrm{~cm}^{-2}$, which agrees with three of the six fitted models shown in Table 2 .

\section{RADIO OBSERVATIONS AND ANALYSIS}

Radio observations were carried out contemporaneously with the XMM-Newton satellite observations. The first radio observations were performed with the 64-m Parkes radio telescope located in NSW, Australia using the multibeam receiver. After PSR J1819-1458 set at Parkes, we continued observing the source with the 100-m Effelsberg radio telescope located in Effelsberg, Germany. Just before the Effelsberg observations ended, we started observing PSR J1819-1458 with the 105-m GBT in Green Bank, WV, USA. The GBT mea- 
surements were followed up once again with the Parkes radio telescope for the remaining hour of the scheduled XMMNewton observations. The durations and parameters of each radio observation are summarized in Table 3 .

Radio pulses were first searched for by dedispersing the GBT and Parkes telescope data both at the dispersion measure of PSR J1819-1458, $196.0 \mathrm{~cm}^{-3} \mathrm{pc}$, and with zero dispersion using the SIGPROC 12 pulsar processing package. Zero-DM time series were created for the GBT and Parkes telescope data to help discriminate pulses from terrestrial radio sources. We could not dedisperse the Effelsberg telescope data because it had only one frequency channel. The radiometer noise, which is the root-mean-square deviation of the time series in flux density units, determines the sensitivity of each observation and is given by

$$
\sigma=\frac{\beta T_{\text {sys }}}{G \sqrt{n_{\mathrm{p}} t_{\mathrm{samp}} \Delta f}},
$$

where $\beta$ is a correction factor accounting for the loss in sensitivity due to digitization $(1.25,1.16$, and 1.00 for one-, three-, and sixteen-bit digitization of the Parkes, GBT, and Effelsberg Telescopes, respectively), $T_{\text {sys }}$ is the system temperature (we included scaled 408-MHz sky temperatures of Haslam et al. (1981) assuming a spectral index of -2.6 (Lawson et al. 1987) in the values quoted in Table 3), $G$ is the gain, $n_{\mathrm{p}}$ is the number of polarizations summed (two in our case), $t_{\text {samp }}$ is the sampling time, and $\Delta f$ is the bandwidth of the observation. The parameters for each observation are detailed in Table 3 . The effective sampling time of the Effelsberg telescope of $46 \mathrm{~ms}$ listed in Table 3 is the dispersion delay of the pulse over the single frequency channel's bandwidth of its receiver. Since this effective sampling time makes the Effelsberg telescope's radiometer noise misleadingly lower, we also provide a modified radiometer noise for comparison, $\sigma_{1 \mathrm{~ms}}$, which uses $t_{\text {samp }}=1 \mathrm{~ms}$. Radio pulses which were detected with higher SNR at the DM of the source than at zero DM (for the GBT and Parkes telescope data), exceeded the radiometer noise by a factor of five considering the false-alarm statistics, and were in phase with the radio ephemeris were considered real.

The times of arrival of the pulses from the RRAT were converted to barycentered arrival times at infinite frequency using TEMPO and the X-ray derived position. The folded solar system barycentered times are shown in the middle panel of Figure 1, which was created by finding the phase of each pulse using the radio ephemeris and then binning all the radio pulse arrival times into a 2048 bin histogram. While individual radio pulses of PSR J1819-1458 typically consists of a single narrow pulse, the averaged radio pulse shape, shown in the bottom panel of Figure 1, has three separate components (Lyne et al. 2009; Karastergiou et al. 2009), a center component of more fainter pulses and two outer components made of fewer brighter pulses. Each outer component is $\sim 45 \mathrm{~ms}$ apart from the center component, much smaller than one of the ten bins in the top panel of Figure 1 and does not affect the correlation analysis in Section 4 since only correlations greater than one spin period are considered. We detected 165 radio pulses in the first Parkes observation (i.e. 21 pulses/hour), 64 pulses in the Effelsberg observation (i.e. 12 pulses/hour), 673 pulses in the GBT observation (i.e. 90 pulses/hour), and 29 pulses in the second Parkes observation (i.e. 29 pulses/hour) for 931 radio pulses (bottom panel of Figure 3 ).

\footnotetext{
12 http://sigproc.sourceforge.net
}

\section{CORRELATION OF RADIO PULSES AND X-RAY PHOTONS}

For the correlation analysis we only considered X-ray photons from the $0.5-2.6 \mathrm{keV}$ energy range determined in Section 2.1. shown as the dashed line in Figure 3. Analysis of the X-ray events within the GTIs satisfying either PATTERN $=0$ (i.e. allowing for only single events) as well as PATTERN $\leq 12$ was performed to see if this had any effect on the result. The PATTERN $=0$ requirement, not shown, yielded 4166 PN events, 1425 MOS1 events, and 1512 MOS2 events for a total of 7103 detections. and PATTERN $\leq 12$ requirement, shown in Figure 4 yielded 5692 PN events, 1705 MOS1 events, and 1767 MOS2 events for a total of 9164 detections.

The radio coverage described in Section 3 was not continuous due to two gaps - one between the first Parkes and Effelsberg observation and one between the GBT and second Parkes observation. The second Parkes telescope observation was contemporaneous with our XMM-Newton observation, but that portion of the XMM-Newton data was completely excluded by the GTIs. Due to the discontinuous radio and X-ray observation coverage as well as differences in radio telescope sensitivities, the distribution of time delays between X-ray detections and radio pulse detections is non-Gaussian. In order to measure the significance of any correlations between detected radio pulses and X-ray photons from PSR J1819-1458, we created a series of random X-ray photon distributions that would be consistent with the discontinuous coverage. We distributed the photon times throughout the GTIs, sampling from a flat (random) distribution. We created an array of $10^{4}$ random X-ray distributions to then compare to the radio pulse arrival times, in addition to the comparison with the XMMNewton data.

We calculated the number of X-ray events detected by the $\mathrm{PN}$ and MOS cameras coincident with detected radio pulses at different lag times (see Figure 4). The lag time for each $\mathrm{X}$-ray photon was calculated as the time elapsed between the $\mathrm{X}$-ray detection time and its nearest detected radio pulse, either before or after the X-ray detection. In this case, an X-ray detection was considered coincident if there was a radio pulse detected within some specified window of time, e.g. for a window of ten periods an X-ray photon was counted as coincident if there was at least one radio pulse detected within $10 \times 4.26 \mathrm{~s}=42.6 \mathrm{~s}$ of either before or after the X-ray event. This was also done for the array of simulated random sets. Then the mean and standard deviation of these sets for each lag window were calculated (the squares with vertical error bars in Figure 4). Differences between the data and simulated random sets are shown on the middle plot of Figure $4 . \mathrm{Fi}$ nally, the differences were divided by the standard deviations of the simulated random sets, shown in the bottom plot of Figure 4. For most lag window sizes, the number of coincident $\mathrm{X}$-ray photons in the data exceeds the number of coincident $\mathrm{X}$-ray photons in the simulated random sets. The largest deviation between the data and the simulations is $3.2 \sigma$ and $3.4 \sigma$ at $3 P \approx 12.8 \mathrm{~s}$ (where $P$ is the rotational period of the pulsar) for the PATTERN $=0$ and PATTERN $\leq 12$ cases, respectively. Specifically, there were 1352 coincident X-ray detections but a mean of only 1262 coincident photons from the simulated random sets with a standard deviation of 32 photons with a $3 P$ window size for the PATTERN $=0$ case and 1742 coincident X-ray detections but only a mean of 1617 coincident photons from the simulated random sets with a standard deviation of 36 photons with a $3 P$ window size for the PATTERN $\leq 12$ case. Of our $10^{4}$ random sets, only 46 sets had a deviation 
exceeding $3.4 \sigma$ for one or more window sizes, the probability of this occurring by chance is then $0.46 \%$. Note that as the window size gets large enough, the data and simulated data sets converge once all the photons are considered coincident.

To help us gauge the significance of these deviations of the data versus randomized times, we also did the same analysis for another source on the same CCD in the field of view of XMM-Newton, 2XMMi J181928.8-145202 ${ }^{13}$, (see Figure 5). This source was not visible on the MOS1 detector, but we only considered the PN detector for this comparison. In this case, the randomized times are coincident more often than the real data, with the largest deviation peaking at $2.7 \sigma$ below the mean of the simulations at $10 P=42.63 \mathrm{~s}$. Of these $10^{4}$ random sets, 408 sets had a deviation exceeding $2.7 \sigma$ for one or more window sizes, the probability of this occurring by chance is then $4.08 \%$.

The Kolmogorov-Smirnov (KS) test (see, e.g., Press et al. 1986 ) was used to determine the degree to which the X-ray data set itself differs from a random distribution. In this case we used both the numerical recipes ksone, which compares a single data set to an analytical distribution, and kstwo, which compares two data sets to one another. When comparing the combined PN and MOS detections to a flat distribution throughout the GTIs, the KS statistic from ksone is 0.14 (note that small values indicate the set is significantly different from the distribution). When we compared our $10^{4}$ simulated random sets to the distribution with $\mathrm{ks}$ one, we found a mean KS statistic of $0.5 \pm 0.3$. We also compared the PN and MOS detections to the array of simulated random sets using kstwo. In this case, the mean KS statistic of these comparisons is $0.3 \pm 0.3$, where the \pm 0.3 represents the standard deviation of the $10^{4}$ cases. We compared the radio pulse detections at each observation to a random distribution. The KS statistic from ksone was 0.77, 0.07, 0.09, and 0.23 for the first Parkes, Effelsberg, GBT, and second Parkes observations, respectively. We then compared each observation's pulse detections to $10^{4}$ simulated random sets with flat distributions containing the same number of pulse detections using kstwo. The mean KS statistic of these comparisons is $0.6 \pm 0.3,0.5 \pm 0.3,0.5 \pm 0.3$, and $0.5 \pm 0.3$ for the first Parkes, Effelsberg, GBT, and second Parkes observations, respectively. These statistics show that individually the X-ray photons and the radio pulse detections are consistent with random distributions.

\section{CONCLUSIONS}

We have observed concurrent X-ray and radio pulsations from PSR J1819-1458. The peak of the X-ray profile is offset from the radio profile by $0.02 \pm 0.01$ in phase, which means they occur at the same phase within the timing resolution of $X M M$-Newton (73.4 ms, or 0.017 of the period). There is also evidence of a second sine-wave at twice the rotational frequency of the radio pulses and aligned with the X-ray profile peak, suggesting X-ray emission from the other pole of the neutron star and befitting a two blackbody model with both poles as hotspots. This is consistent with radio polarization observations which show that PSR J1819-1458 could be an orthogonal rotator (the angle between the pulsar's rotational axis and its magnetic dipole axis, $\alpha$, is not well-constrained, but most likely has a value near $90^{\circ}$, see Karastergiou et al. (2009)).

The spectrum is consistent with a thermal emitter with a broad absorption line, possibly composed of two different

\footnotetext{
${ }^{13}$ http://xmmssc-www.star.le.ac.uk/Catalogue/2XMMi/
}

lines around $\sim 1.0$ and $\sim 1.3 \mathrm{keV}$. Coupled with the detection of the absorption seen in a previous XMM-Newton observation (McLaughlin et al. 2007) and in the Chandra data (Rea et al. 2005; Camero-Arranz et al. 2012), we are certain of its astrophysical nature. If the line is due to proton resonant cyclotron scattering, then the cyclotron absorption line at $0.907 \mathrm{keV}(\mathrm{BB} \times \mathrm{Cyc}$ lot ron in Table 2) implies a dipole magnetic field strength of $1.9 \times 10^{14} \mathrm{G}$. If the absorption line is due to electron resonant cyclotron scattering, then the dipole magnetic field strength would be closer to $1 \times 10^{11} \mathrm{G}$. The surface dipole magnetic field strength estimate is proportional to the cosecant of $\alpha$ (Lorimer $\&$ Kramer 2005). The surface dipole magnetic field strength is then consistent with the cyclotron proton resonant scattering model for $\alpha=15^{\circ}$. As we have described above there is evidence that $\alpha$ may be closer to $90^{\circ}$, though $\alpha$ is not well constrained. The inferred $\alpha$ for the electron cyclotron case is undefined since the implied dipole magnetic field strength of the model would be weaker than the surface dipole magnetic field strength estimate. This makes the electron cyclotron model unlikely.

We fit a blackbody temperature of $k T \sim 0.14 \mathrm{keV}$, slightly higher than what is expected from fast cooling models for high magnetic field pulsars (Aguilera et al. 2008; Pons et al. 2009). This relation, however, assumes the spin-down age to be correct, which might not be true (Noutsos et al. 2013), especially given the unusual glitch behavior of PSR J1819-1458 (Lyne et al.2009). It is also interesting to consider that the derived X-ray luminosity from our best-fit model, a blackbody model with two Gaussian absorption lines, is $L_{0.3-5.0 \mathrm{keV}} \sim$ $3 \times 10^{33} \mathrm{ergs} \mathrm{s}^{-1}$, which exceeds the pulsar's spin-down luminosity by a factor of $\sim 10$. The $>25 \%$ uncertainty in the distance estimate, however, lends an even larger uncertainty to the derived X-ray luminosity estimate. The temperature and possibly high luminosity, combined with the unusual glitch activity, suggests that it could be a transitional object between pulsars and magnetars.

Our KS test results show that both the X-ray photon and radio pulse detections are consistent with random distributions. However, we have shown that the X-ray photon and radio pulse detections may be correlated on timescales of less than 10 pulsar spin periods, where we measured a $3.4 \sigma$ deviation in our data from random distributions. As mentioned in Section 1 this tentative correlation suggests a link between the physical process producing the radio pulses and the heating of the polar-cap and represents the first enhancement of $\mathrm{X}$-ray emission associated with radio pulse variability.

Zhang et al. (2007) proposed two interpretations which may explain the relationship between nulling pulsars, RRATs, and conventional radio pulsars. Their first model interpreted RRATs and nulling pulsars as dead pulsars which sporadically re-activate when coherent emission and pair production conditions are met. Their second model interpreted RRATs' behavior as a complement to nulling pulsars undergoing a reversal of radio emission direction. Zhang et al. proposed that X-ray observations may help discern between the two interpretations and specifically mention PSR J1819-1458 as fitting within the re-activated dead pulsar model because of its apparent lack of a non-thermal component in its X-ray spectrum (Reynolds et al. 2006; Gaensler et al. 2007). Even though we are currently unable to constrain a power-law tail, the tentative correlation between the radio pulse and X-ray photon detection times suggests the reactivation model for PSR J1819-1458. 
The National Radio Astronomy Observatory is a facility of the National Science Foundation operated under cooperative agreement by Associated Universities, Inc. JM was supported by an NRAO student support grant. JM and MAM were supported by NASA XMM-Newton observer support award NNX10AD14G. MAM gratefully acknowledges support from Oxford Astrophysics while on sabbatical leave. NR acknowledges support from a Ramon y Cajal fellowship and grants AYA2009-07391, AYA2012-39303, SGR2009-811, TW2010005 and iLINK2011-0303.

\section{REFERENCES}

Aguilera, D. N., Pons, J. A., \& Miralles, J. A. 2008, A\&A, 486, 255

Bilous, A. V., Kondratiev, V. I., McLaughlin, M. A., Ransom, S. M., Lyutikov, M., Mickaliger, M., \& Langston, G. I. 2011, ApJ, 728, 110 Camero-Arranz, A., et al. 2012, ArXiv e-prints

Cordes, J. M., \& Lazio, T. J. W. 2002, astro-ph/0207156

Cordes, J. M., \& Shannon, R. M. 2008, ApJ, 682, 1152

Dickey, J. M., \& Lockman, F. J. 1990, Ann. Rev. Astron. Astrophys., 28, 215

Esamdin, A., Zhao, C. S., Yan, Y., Wang, N., Nizamidin, H., \& Liu, Z. Y. 2008, MNRAS, 389, 1399

Gaensler, B. M., et al. 2007, Ap\&SS, 308, 95

Garmire, G. P., Bautz, M. W., Ford, P. G., Nousek, J. A., \& Ricker, Jr., G. R. 2003, in Society of Photo-Optical Instrumentation Engineers (SPIE) Conference Series, Vol. 4851, Society of Photo-Optical Instrumentation Engineers (SPIE) Conference Series, ed. J. E. Truemper \& H. D. Tananbaum, 28-44

Haslam, C. G. T., Klein, U., Salter, C. J., Stoffel, H., Wilson, W. E., Cleary, M. N., Cooke, D. J., \& Thomasson, P. 1981, Astron. Astrophys., 100, 209 He, C., Ng, C.-Y., \& Kaspi, V. M. 2013, ApJ, 768, 64

Hohle, M. M., Haberl, F., Vink, J., de Vries, C. P., \& Neuhäuser, R. 2012, MNRAS, 419, 1525

Jenet, F. A., \& Anderson, S. B. 1998, Publ. Astron. Soc. Pac., 110, 1467

Kalberla, P. M. W., Burton, W. B., Hartmann, D., Arnal, E. M., Bajaja, E., Morras, R., \& Pöppel, W. G. L. 2005, A\&A, 440, 775

Kaplan, D. L., \& van Kerkwijk, M. H. 2011, ApJ, 740, L30

Karastergiou, A., Hotan, A. W., van Straten, W., McLaughlin, M. A., \& Ord, S. M. 2009, MNRAS, 396, L95

Kargaltsev, O., Durant, M., Misanovic, Z., \& Pavlov, G. G. 2012, Science, 337,946

Keane, E. F., \& McLaughlin, M. A. 2011, Bulletin of the Astronomical Society of India, 39, 333
Lawson, K. D., Mayer, C. J., Osborne, J. L., \& Parkinson, M. L. 1987, Mon. Not. R. Astron. Soc., 225, 307

Lodders, K. 2003, ApJ, 591, 1220

Lorimer, D. R., \& Kramer, M. 2005, Handbook of Pulsar Astronomy (Cambridge University Press)

Luo, Q., \& Melrose, D. 2007, MNRAS, 378, 1481

Lyne, A. G., McLaughlin, M. A., Keane, E. F., Kramer, M., Espinoza, C. M., Stappers, B. W., Palliyaguru, N. T., \& Miller, J. 2009, MNRAS, 400, 1439

McLaughlin, M. A., et al. 2006, Nature, 439, 817

-. 2007, ApJ, 670, 1307

Noutsos, A., Schnitzeler, D., Keane, E., Kramer, M., \& Johnston, S. 2013, ArXiv e-prints

Pons, J. A., Miralles, J. A., \& Geppert, U. 2009, A\&A, 496, 207

Press, W. H., Flannery, B. P., Teukolsky, S. A., \& Vetterling, W. T. 1986, Numerical Recipes: The Art of Scientific Computing (Cambridge: Cambridge University Press)

Rea, N., Oosterbroek, T., Zane, S., Turolla, R., Méndez, M., Israel, G. L. Stella, L., \& Haberl, F. 2005, MNRAS, 361, 710

Rea, N., et al. 2009, ApJ, 703, L41

Reynolds, S. P., et al. 2006, ApJ, 639, L71

Shearer, A., Stappers, B., O’Connor, P., Golden, A., Strom, R., Redfern, M., \& Ryan, O. 2003, Science, 301, 493

Turolla, R. 2009, in Astrophysics and Space Science Library, Vol. 357, Astrophysics and Space Science Library, ed. W. Becker, 141

Watson, M. G., et al. 2009, A\&A, 493, 339

Weisskopf, M. C., Brinkman, B., Canizares, C., Garmire, G., Murray, S., \& Van Speybroeck, L. P. 2002, Publ. Astron. Soc. Pac., 114, 1

Yakovlev, D. G., \& Pethick, C. J. 2004, Ann. Rev. Astron. Astrophys., 42, 169

Zhang, B., Gil, J., \& Dyks, J. 2007, MNRAS, 374, 1103 


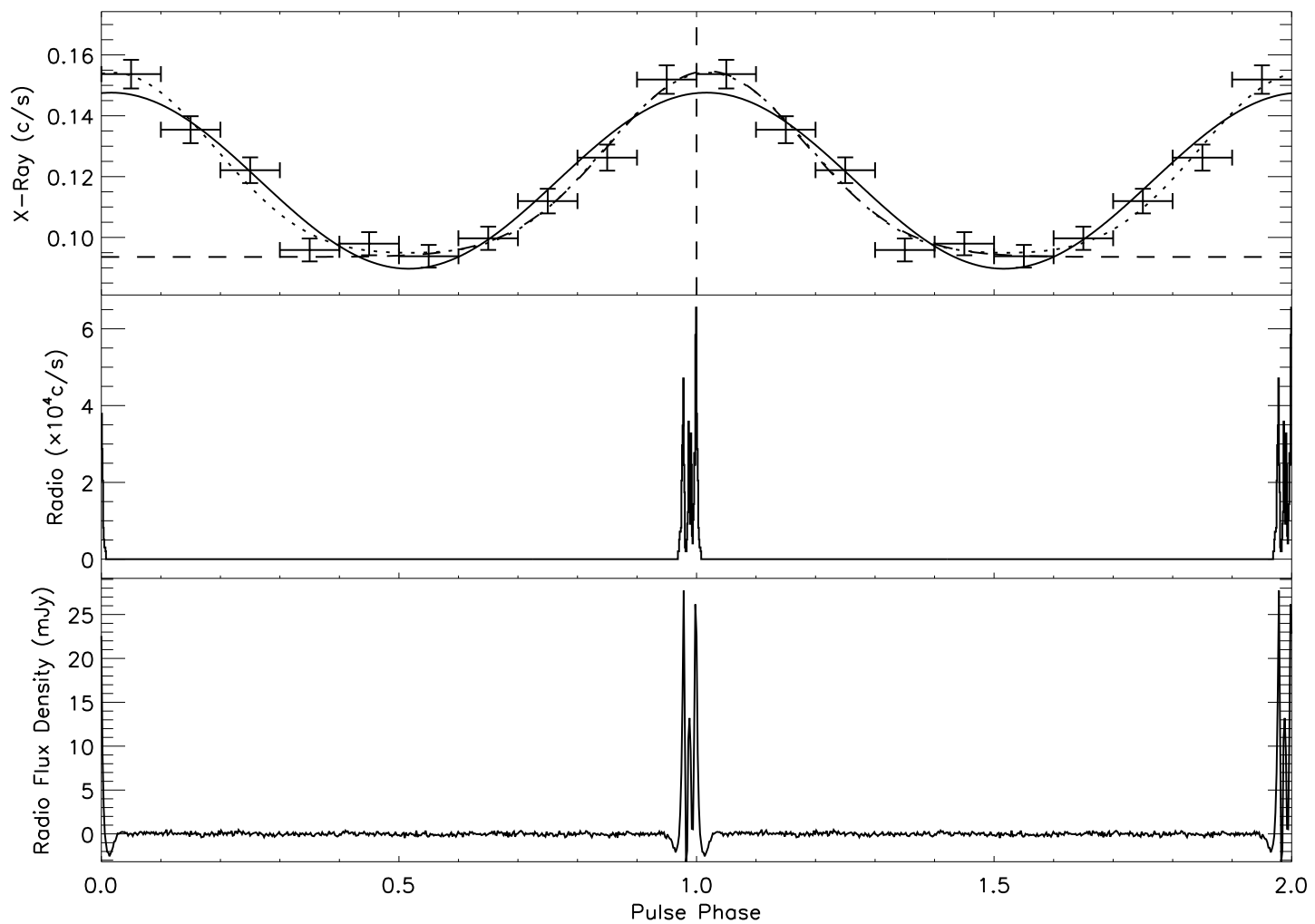

FIG. 1.- X-ray and radio profiles of PSR J1819-1458 folded using the radio ephemeris. Top: The background-corrected X-ray profile consists of ten phase bins over one rotational period, which consists of XMM-Newton PN and MOS detected photons within the $0.5 \mathrm{keV}<E<2.6 \mathrm{keV}$ energy range within the GTIs and PATTERN $\leq 12$, summing up to $\sim 17$ hours of observation time. The horizontal and vertical bars indicate the size of the phase bins and the $\sqrt{N}$ errors. The solid, dotted, and dashed lines indicate the single sinusoid, two sinusoid, and Gaussian fits to the profile (fit over the $0.5-1.5$ phase range), respectively. Note that the dotted and dashed lines overlap considerably. The vertical dashed line indicates the peak of the radio pulse profile (phase $=1.0$ ). Middle: Radio pulse count histogram created by using the radio ephemeris to assign a phase to each barycentered pulse detected by the 7.7 hour observation of the GBT at an observing frequency of $2 \mathrm{GHz}$, and then binning all the radio pulse arrival times into a 2048 bin histogram. Bottom: Radio flux density profile formed from pulses detected using the 7.7 hour observation of the GBT at an observing frequency of $2 \mathrm{GHz}$. Flux densities were calculated by normalizing the scale of each detected pulse's off-pulse noise to the radiometer noise, then averaging all the pulses together. The dips preceding and following the pulse are due to digitization of the signal (e.g. Jenet \& Anderson 1998). The profile is shown twice in all plots for clarity.

TABLE 1

X-RAY GOOD TIME INTERVALS

\begin{tabular}{lccc}
\hline \hline & PN & MOS1 & MOS2 \\
\hline GTI 1 MJD span & $54556.8164062-54556.8496346$ & $54556.8164062-54556.8494625$ & $54556.8164062-54556.8503720$ \\
GTI 2 MJD span & $54556.8496771-54556.8504032$ & $54556.8496430-54556.8503653$ & $54556.8505225-54557.6338842$ \\
GTI 3 MJD span & $54556.8505407-54557.6093750$ & $54556.8505158-54557.6093750$ & $54557.6339443-54557.6093750$ \\
\hline
\end{tabular}

NOTE. - Good Time Intervals (GTIs) for the PN, MOS1, and MOS2 detectors. See Section 2 for details. 


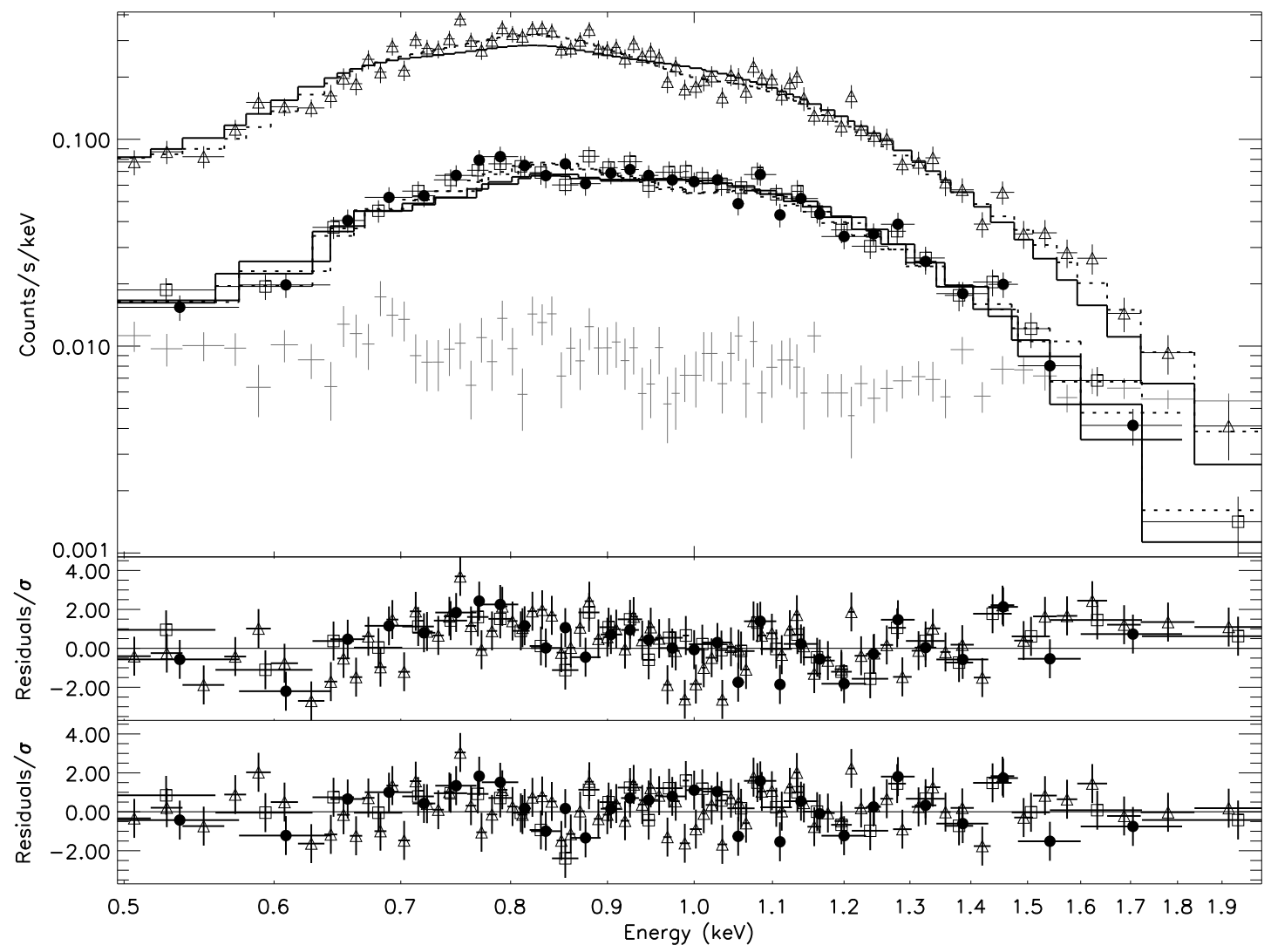

FIG. 2.- X-ray spectrum of PSR J1819-1458 using photons with energies in the $0.5-2.0 \mathrm{keV}$ range and PATTERN $\leq 4$ from both our observation and McLaughlin et al. (2007). Data have been rebinned for plotting purposes by a factor of two from 157, 63, and 66 bins to 78, 30, and 33 bins for PN, MOS1, and MOS2, respectively. Top: The dark crosshairs indicate the PN (triangles), MOS1 (filled circles) and MOS2 (squares) source spectra, respectively. The light crosshairs represent the PN background spectrum. The solid lines indicate the simplest model fit, a blackbody with interstellar absorption, an underabundance of oxygen, and solar abundances from Lodders (2003) for elements other than hydrogen and oxygen (vphabs $*$ bbody); while the dotted lines indicate one of the best model fits (vphabs*gabs*gabs*bbody), which also includes two Gaussian absorption lines around 1.0 and $1.3 \mathrm{keV}$. Middle: Normalized PN (triangles), MOS1 (filled circles) and MOS2 (squares) residuals for the vphabs *bbody model. Bottom: Normalized PN (triangles), MOS1 (filled circles) and MOS2 (squares) residuals for the vphabs*gabs*gabs $*$ bbody model. 


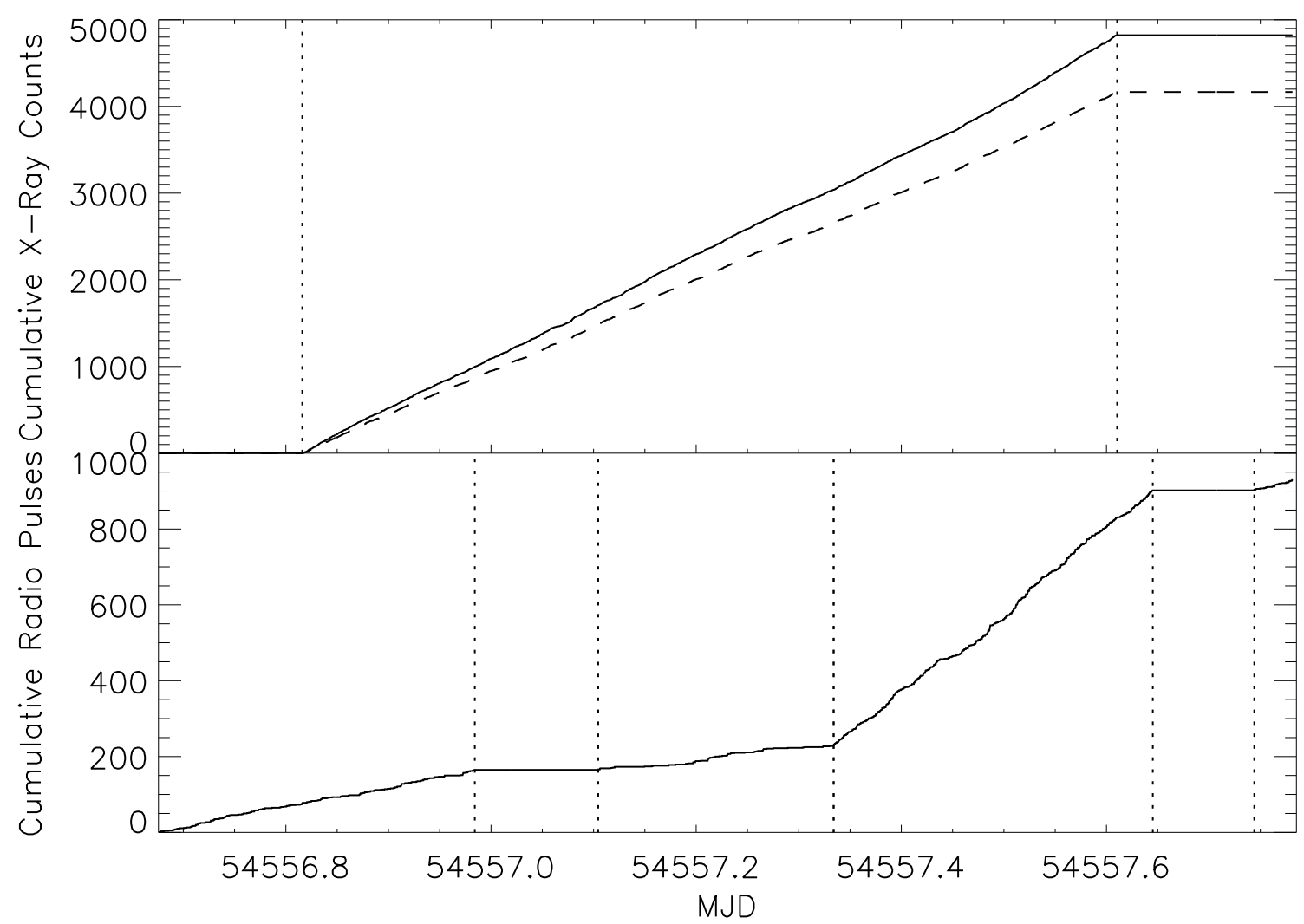

FIG. 3.- Top: Cumulative count of X-ray photon detections from PSR J1819-1458. The solid line represents all X-ray photons while the dashed only includes the photons with energies within the range $0.5-2.6 \mathrm{keV}$. Dashed vertical lines designate the beginning and end of the GTIs. In both cases, we only include photons from the source region described in Section 2.1 Bottom: Cumulative radio pulses detected by the following radio telescopes over time - Parkes, Effelsberg, GBT, and then Parkes again. Dashed vertical lines indicate the beginnings and endings of the radio telescopes' observing time. Different radio observing frequencies and sensitivities bring about the different slopes of the cumulative radio pulse distribution. The two flat regions of the distribution are attributed to the times when the pulsar was not observed. 


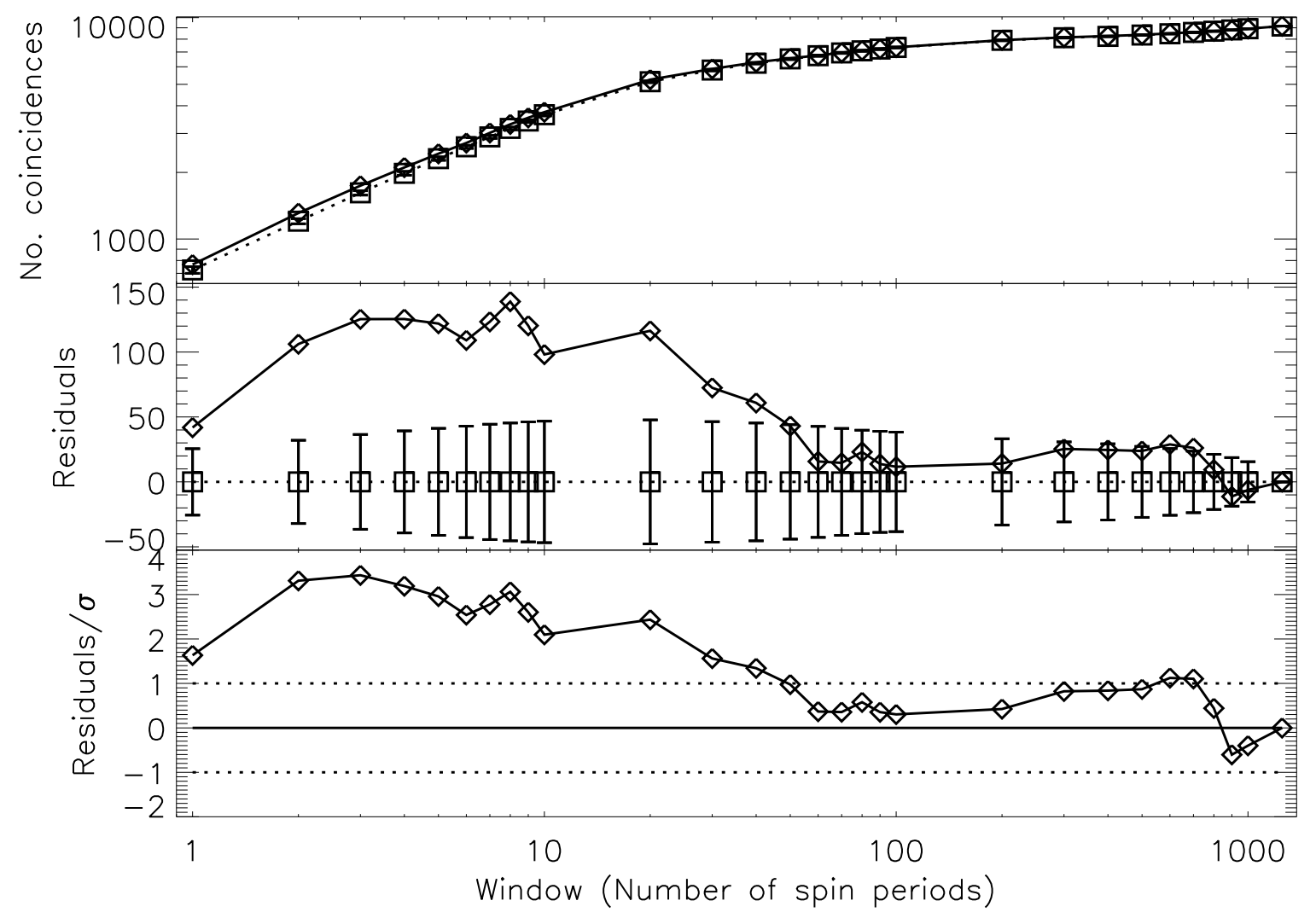

FIG. 4.- Top: Comparison of the number of PATTERN $\leq 12$ X-ray photons from PSR J1819-1458 within the 0.5-2.6 keV energy range of the PN and MOS cameras that are coincident with a radio pulse within a given search window. X-ray photons coincident with the radio pulses are represented by diamonds and a solid line while the results of our simulation are represented by the squares (mean coincident photons of the simulations), vertical bars (standard deviation of coincident photons of the simulations) and the dotted line. Middle: The difference in the number of coincident photons for each window size in the data and the mean of the simulations. Bottom: As the middle plot, normalized by the standard deviation of the simulations. Here the horizontal dotted line indicates one standard deviation of the simulated random sets. 


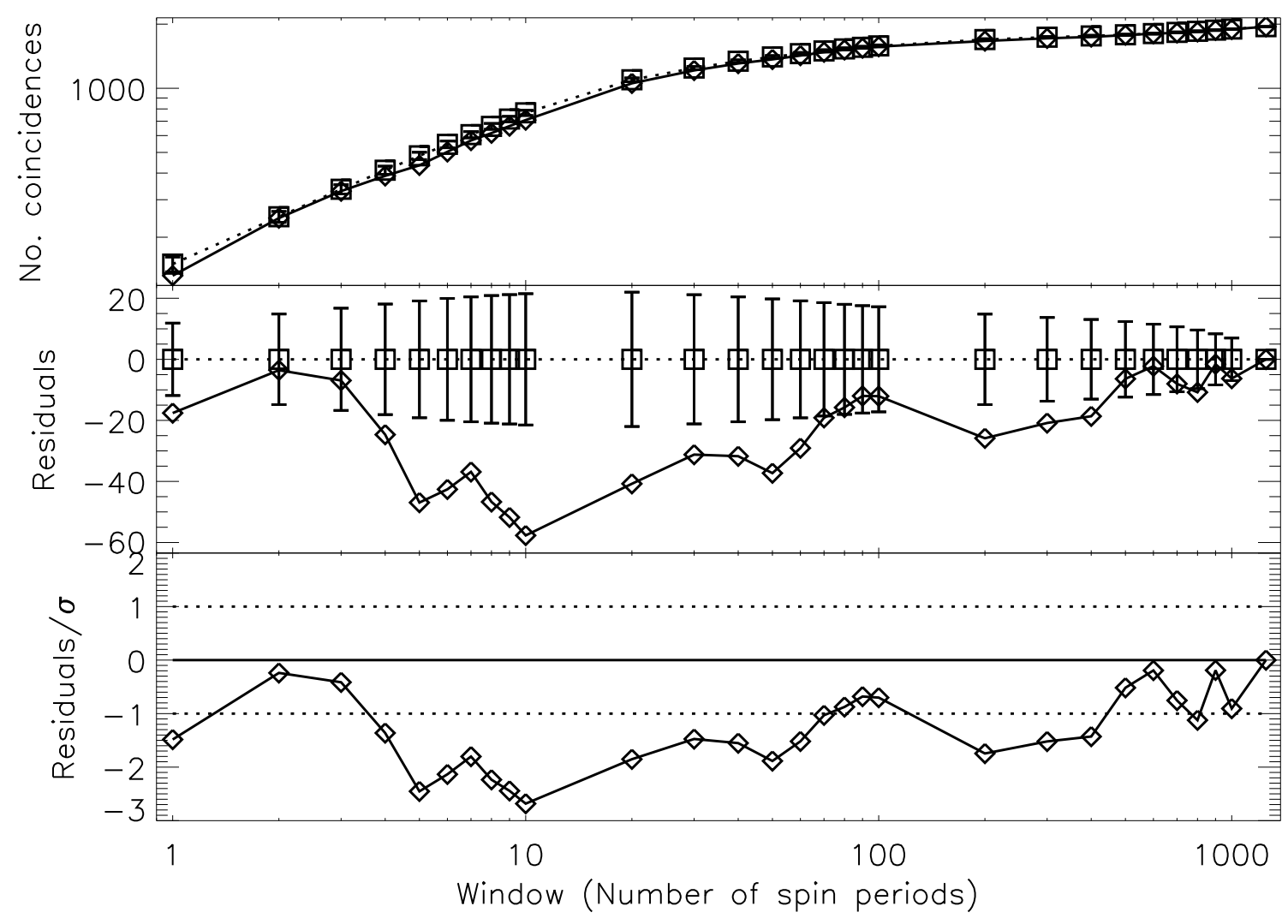

FIG. 5.- Top: Comparison of the number of X-ray photons from 2XMMi J181928.8-145202 within the 0.5-2.6 keV energy range from the PN detector only that are coincident with a radio pulse within a given search window. X-ray photons coincident with the radio pulses are represented by diamonds and a solid line while the results of $10^{4}$ simulations are represented by the boxes (mean coincident photons of the simulations), vertical bars (standard deviation of coincident photons of the simulations) and the dotted line. Middle: The difference between the number of coincident photons for each window size in the data and the mean of the simulations. Bottom: As in the middle plot, normalized by the standard deviation of the simulations. Here the horizontal dotted line indicates one standard deviation of the simulated random sets. 
TABLE 2

SPECTRAL FITS FOR PSR J1819-1458 WITH EPIC-PN

\begin{tabular}{|c|c|c|c|c|c|c|}
\hline & Blackbody (BB) & $\mathrm{BB} \times \mathrm{Neon}$ & $\mathrm{BB} \times$ Gaussian & $\mathrm{BB} \times$ Cyclotron & $\mathrm{BB}+\mathrm{BB}$ & $\mathrm{BB} \times$ Gaussian $\times$ Gaussian \\
\hline$N_{\mathrm{H}}$ & $0.9 \pm 0.1$ & $0.88_{-0.08}^{+0.10}$ & $1.244 \pm 0.009$ & $1.174 \pm 0.009$ & $1.4_{-0.2}^{+0.3}$ & $0.88 \pm 0.01$ \\
\hline$N_{O}$ & $0.3_{-0.3}^{+0.2}$ & $0.7_{-0.3}^{-0.08}$ & $0.82 \pm 0.02$ & $0.87 \pm 0.02$ & $0.8_{-0.2}^{+0.1}$ & $0.69 \pm 0.03$ \\
\hline$N_{\mathrm{Ne}}$ & - & $3.0 \pm 0.7$ & - & - & - & - \\
\hline$E_{\mathrm{cy}}$ & - & - & - & $0.907 \pm 0.009$ & - & - \\
\hline$w_{\mathrm{cy}}$ & - & - & - & $0.54 \pm 0.02$ & - & - \\
\hline$d_{\mathrm{cy}}$ & - & - & - & $1.32 \pm 0.02$ & - & - \\
\hline$E_{\mathrm{G} 1}$ & - & - & $1.12 \pm 0.01$ & - & - & $1.00 \pm 0.01$ \\
\hline$\sigma_{\mathrm{G} 1}$ & - & - & $0.39 \pm 0.01$ & - & - & $0.004 \pm 0.001$ \\
\hline$\tau_{\mathrm{G} 1}$ & - & - & $1.41 \pm 0.03$ & - & - & $4_{-3}^{+51}$ \\
\hline$E_{\mathrm{G} 2}$ & - & - & - & - & - & $1.29 \pm 0.03$ \\
\hline$\sigma_{\mathrm{G} 2}$ & - & - & - & - & - & $0.18 \pm 0.03$ \\
\hline$\tau_{\mathrm{G} 2}$ & - & - & - & - & - & $0.18 \pm 0.02$ \\
\hline$k T_{1}$ & $0.140 \pm 0.005$ & $0.131 \pm 0.006$ & $0.1133 \pm 0.0005$ & $0.1312 \pm 0.0007$ & $0.07 \pm 0.01$ & $0.1382 \pm 0.0009$ \\
\hline$k T_{2}$ & - & - & - & - & $0.15_{-0.01}^{+0.02}$ & - \\
\hline Abs. Flux & $1.35_{-0.03}^{+0.02}$ & $1.36_{-0.05}^{+0.02}$ & $1.37 \pm 0.03$ & $1.37 \pm 0.03$ & $1.37 \pm 0.03$ & $1.37_{-0.04}^{+0.07}$ \\
\hline Unab. Flux 1 & $13.3 \pm 0.3$ & $25.5 \pm 0.5$ & $224 \pm 5$ & $155 \pm 3$ & $520 \pm 20$ & $21.8 \pm 0.5$ \\
\hline Unab. Flux 2 & - & - & - & - & $14.5 \pm 0.7$ & - \\
\hline$R_{1}$ & $6 \pm 4$ & $10 \pm 6$ & $40 \pm 20$ & $30_{-20}^{+10}$ & $100 \pm 100$ & $8_{-4}^{+5}$ \\
\hline$R_{2}$ & - & - & - & - & $6_{-3}^{+4}$ & $\begin{array}{l}-4 \\
-\end{array}$ \\
\hline$\chi_{\nu}^{2}$ (d.o.f.) & $1.41(153)$ & $1.28(152)$ & $1.21(150)$ & $1.19(150)$ & $1.19(151)$ & 1.09 (147) \\
\hline
\end{tabular}

Note. - Parameters fit to our data combined with the McLaughlin et al. (2007) data, fitting in the 0.5-2.0 keV energy range. Fluxes are calculated in the 0.3-5.0 keV energy range for direct comparison to the observation done by McLaughlin et al. (2007), reported in units of $10^{-13} \mathrm{ergs} \mathrm{s}^{-1} \mathrm{~cm}^{-2} . N_{\mathrm{H}}$ is in units of $10^{22} \mathrm{~cm}^{-2}$ while $N_{\mathrm{O}}$ and $N_{\mathrm{Ne}}$ are in solar units (assuming solar abundance from Lodders (2003)). The photoelectric cross-section of Verner et al. (1998) has been used for all fits. The values of $k T$ (blackbody temperature), $E_{\mathrm{G}}$ (Gaussian line energy), $\sigma_{\mathrm{G}}$ (Gaussian line width), $E_{\mathrm{cy}}$ (cyclotron line energy) and $w_{\mathrm{cy}}$ (cyclotron line width) are in units of keV. $R_{1}$ (blackbody emission radius at infinity assuming a $3.6 \mathrm{kpc}$ distance) and $R_{2}$ (blackbody hotspot emission radius at infinity in the two blackbody model, also assuming a $3.6 \mathrm{kpc}$ distance) are in units of $\mathrm{km}$. The Gaussian line depth $\tau_{\mathrm{G}}$ and fundamental cyclotron line depth $d_{\mathrm{cy}}$ are dimensionless. Errors are at the $1 \sigma$ confidence level. XSPEC models used are (from left to right): vphabs $*$ bbody, vphabs $* b b \circ d y$,

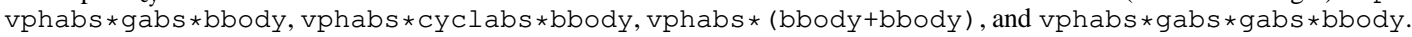

TABLE 3

RADio PARAMETERS

\begin{tabular}{lcccc}
\hline \hline & Parkes 1 & Effelsberg & GBT & Parkes 2 \\
\hline MJD span & $54556.67-54557.00$ & $54557.10-54557.33$ & $54557.33-54557.65$ & $54557.74-54557.78$ \\
Center Freq. (GHz) & 1.4 & 1.4 & 1.9 & 1.4 \\
Bandwidth (MHz) & 256 & 80 & 600 & 256 \\
No. of frequency channels & 512 & 1 & 768 & 512 \\
Sampling Time $(\mu \mathrm{s})$ & 100 & 46000 & 81.92 & 100 \\
Observation Length (hr) & 7.9 & 5.5 & 7.7 & 1.0 \\
$\beta$ & 1.25 & 1.00 & 1.16 & 1.25 \\
$G(\mathrm{~K} / \mathrm{Jy})$ & 0.67 & 1.5 & 1.9 & 0.67 \\
$T_{\text {sys }}(\mathrm{K})$ & 39 & 27 & 29 & 39 \\
$\sigma(\mathrm{mJy})$ & 320 & 4.6 & 56 & 320 \\
$\sigma_{1 \mathrm{~ms}}(\mathrm{mJy})$ & 100 & 45 & 16.2 & 100 \\
\hline
\end{tabular}

NotE. - Radio observation parameters. See Section 3 for details. 